\title{
Codificação de Imagens com Predição Adaptativa Baseada no Critério de Mínimos Quadrados
}

\author{
Danillo Graziosi $^{1,3}$, Nuno M. M. Rodrigues ${ }^{1,2}$, Eduardo A. B. da Silva ${ }^{3}$, \\ Sérgio M. M. de Faria ${ }^{1,2}$, Murilo B. de Carvalho ${ }^{4}$, Vitor M. M. Silva ${ }^{5}$
}

\begin{abstract}
Resumo-Neste artigo, um novo método de predição baseado no critério dos mínimos quadrados foi incorporado ao algoritmo MMP (Multidimensional Multiscale Parser), com o objetivo de melhorar seu desempenho segundo um critério taxa-distorção.

O contexto local é usado para ajustar adaptativamente os coeficientes de predição linear, que incorporam implicitamente as características da textura local. Uma vez que o decodificador é capaz de repetir o procedimento de estimação dos coeficientes, usando os dados reconstruídos, não é necessário enviar qualquer informação extra. Este novo modo de predição linear adaptado ao contexto foi adicionado aos já existentes modos de predição intra e o melhor modo é escolhido através da otimização do critério de taxa-distorção.

Resultados experimentais, apresentados nas últimas seções deste artigo, mostram que o novo modo de predição é capaz de incorporar ganhos consideráveis ao desempenho de codificação do MMP para imagens suaves, se comparado aos métodos estadoda-arte baseados em transformada, sem apresentar qualquer perda de desempenho quando usado em imagens não-suaves.
\end{abstract}

Palavras-Chave-Codificação de imagens, Casamento de padrões, Predição Intra, Critério dos mínimos quadrados, Processamento de Imagens.

Abstract-In this article, a new prediction method based on least-square minimization was added to the MMP (Multidimensional Multiscale Parser) algorithm, in order to improve its ratedistortion performance.

A local context is used to adaptively adjust the linear prediction coefficients, that implicitly embed the local texture characteristics. Since the decoder repeats the same prediction process using the reconstructed data, no extra overhead is needed for signaling the coefficients. This new context-adaptive linear prediction mode was added to the existing intra prediction modes, and the best mode is chosen through rate-distortion optimization.

The experimental results presented in this paper show that the new prediction mode is able to increase considerably MMPs coding performance for smooth images, when compared with state-of-the-art, transform-based methods, and no performance loss was detected, when applied to non-smooth images.

Keywords - Image Coding, Pattern Matching, Intra Prediction, Least Square Minimization, Image Processing.

\section{INTRODUÇÃo}

Codificadores baseados em transformada são considerados o estado-da-arte para compressão de imagens. Eles exploram

\footnotetext{
${ }^{1}$ Instituto de Telecomunicações, Portugal;

${ }^{2}$ ESTG, Instituto Politécnico de Leiria, Portugal;

${ }^{3}$ PEE/COPPE/DEL/EE, Univ. Fed. do Rio de Janeiro, Brazil

${ }^{4}$ TET, Univ. Fed. Fluminense, Brazil;

${ }^{5}$ DEEC, Univ. de Coimbra, Portugal.

E-mails: danillo@1ps.ufrj.br, eduardo@lps.ufrj.br, nuno.rodrigues@co.it.pt, sergio.faria@co.it.pt, murilo@telecom.uff.br, vitor.silva@co.it.pt. Este trabalho foi apoiado pela Fundação Para a Ciência e Tecnologia (FCT), sob o projeto PTDC/EEA-TEL/66462/2006
}

o fato de imagens suaves apresentarem poucos coeficientes significativos em altas freqüências, que após identificados podem ser descartados ou tratados com um passo de quantização mais elevado, permitindo a compressão de imagens estáticas de forma eficiente. Para melhorar ainda mais o desempenho destes algoritmos, novas ferramentas de codificação foram introduzidas. Por exemplo, os modos de predição direcionais do novo padrão de vídeo H.264/AVC [1] comprimem imagens de forma muito eficiente, permitindo que o padrão de vídeo seja utilizado também para codificar imagens estáticas [2].

Porém nem todas as imagens atendem a premissa da suavidade, como é o caso de imagens obtidas através de scanners ou imagens computadorizadas, geralmente contendo textos e imagens misturados. Essas imagens são também conhecidas como imagens compostas. Para este tipo de imagens, um algoritmo recentemente proposto, o MMP [3], apresenta alguns dos melhores resultados de codificação conhecidos na literatura. Ele se baseia em quantização vetorial e buscas em um dicionário adaptativo, usando múltiplas escalas. Porém o desempenho do MMP para imagens suaves ainda estava abaixo dos algoritmos que representam o estado-da-arte em compressão, como o JPEG2000 [4] e o próprio H.264/AVC modo Intra, no perfil High [1].

Com a introdução de um esquema de predição semelhante ao usado pelo H.264/AVC [5], uma adaptação mais eficiente do dicionário [6] e técnicas adaptativas de segmentação mais flexíveis [7], o MMP agora consegue superar o desempenho taxa-distorção de algoritmos como o JPEG2000 e o H.264/AVC. Porém os modos de predição do H.264/AVC usados também pelo MMP partem do pressuposto que que os valores dos pixels na direção escolhida não irão variar, o que não é necessariamente verdade, especialmente em imagens menos homogêneas. Algumas tentativas de melhorar esse esquema de predição já foram propostas. Em [8] usa-se DPCM nos modos de predição intra para codificação sem perdas, e em [9] os modos de predição são alterados para reutilizarem valores recém-estimados. Predições utilizando casamento de padrões (também conhecidos como template matching) já foram propostas [10], inclusive sendo incorporados ao MMP [11], mostrando que ainda há espaço para melhorias no esquema de predição utilizado atualmente.

Neste artigo propomos adicionar um novo método de predição baseado no critério dos mínimos quadrados (LeastSquare Prediction - LSP). O modo de predição LSP já foi aplicado com sucesso em técnicas de codificação de vídeo [12] e codificação de imagens [13], além de também ter sido implementado para predição em blocos [14]. A predição é feita 
a partir de uma combinação linear dos valores da vizinhança. Os coeficientes utilizados são obtidos por um processo de treinamento local, com base nos valores previamente reconstruídos, portanto não é necessário mandar qualquer informação extra.

Este artigo está organizado da seguinte maneira. A Seção II irá descrever brevemente o algoritmo MMP. O modo de predição baseado no critério dos mínimos quadrados, bem como a sua adaptação para predição em blocos de tamanhos variados, serão apresentados na Seção III. Os resultados serão apresentados na Seção IV, e a Seção V irá concluir o trabalho.

\section{O Algoritmo MMP}

O algoritmo MMP divide a imagem em blocos que não se sobrepoem e usa padrões de um dicionário em diferentes escalas para aproximar os blocos da imagem. Se nenhum padrão presente no dicionário aproxima o bloco da imagem de forma satisfatória, este é então iterativamente dividido, primeiro na vertical, em seguida na horizontal, até atingirmos uma escala onde temos um casamento apropriado entre o bloco e um padrão presente no dicionário. A medida que a imagem vai sendo codificada, os novos padrões que vão surgindo são adicionados ao dicionário em múltiplas escalas, fazendo com que o mesmo se adapte às características da imagem.

A segmentação do bloco e os índices escolhidos são determinados através de um algoritmo baseado no custo Lagrangeano. A melhor segmentação do bloco é escolhida em um passo de otimização, que irá comparar os custos de cada segmento do bloco, e escolher a segmentação que resulta no custo mínimo. O fluxo binário de saída é gerado a partir da codificação aritmética das flags de segmentação seguidas pelos índices dos dicionários escolhidos.

Maiores detalhes sobre o algoritmo MMP e sobre a otimização da taxa-distorção encontram-se em [3].

\section{A. MMP com Predição e Partição Flexível}

Com a finalidade de melhorar o desempenho do MMP para imagens suaves, um passo de predição foi adicionado ao algoritmo de codificação. Desta forma, o algoritmo primeiro realiza a predição do bloco, para em seguida codificar o resíduo utilizando o MMP. O passo de predição é feito de forma hierárquica em conjunto com a segmentação do bloco, e se o mesmo for aplicado de forma eficaz, os resíduos resultantes serão homogêneos e de baixa energia, favorecendo a adaptação do dicionário e melhorando sua eficiência na codificação.

Os modos de predição utilizados foram baseados nos modos presentes no padrão de codificação de vídeo H.264/AVC, porém com tamanhos adaptativos, que devido à segmentação da imagens podem variar desde $16 \times 16$ até $4 \times 4$. O modo de predição é escolhido de acordo com o seu custo Lagrangeano, isto é, de acordo com o custo necessário para transmitir o modo de predição somado ao custo de codificação do resíduo utilizando o MMP. Para cada modo, o custo é calculado e o modo escolhido é o modo que resulta no custo mínimo, representando o melhor compromisso entre a precisão da predição e a taxa extra necessária para enviar este modo.
Desde a introdução do MMP com predição, novos ganhos também foram atingidos com [6]: a redução da entropia dos símbolos do dicionário através da separação dos índices pelo contexto da escala do bloco que originou o novo padrão, a limitação da inserção de elementos novos em determinadas escalas e melhorias no processo de adaptação do dicionário.

Em [15] um novo método de segmentação mais flexível foi proposto (MMP-FP, flexible partition). A partição diádica rígida do MMP foi flexibilizada, e mais uma flag foi introduzida, indicando a direção de segmentação (horizontal ou vertical), de acordo com um melhor compromisso taxadistorção. Esta flexibilização da segmentação concedeu ao MMP ainda um maior poder de adaptação, fazendo com que seu desempenho taxa-distorção ultrapassasse codificadores estado-da-arte para imagens suaves, em taxas médias e altas. A mesma técnica não causou impacto algum no já excelente desempenho que o MMP tem para imagens compostas. Mais detalhes e resultados sobre o MMP-FP podem ser encontrados em [7].

\section{MMP COM PREDIÇÃO BASEADA NO CRITÉRIO DOS MÍNIMOS QUADRADOS}

Como já mencionado anteriormente, o algoritmo MMP-FP utiliza os mesmos modos de predição definidos pelo padrão de codificação de vídeo H.264. A predição irá gerar valores de resíduos que possuem uma função de distribuição de probabilidade concentrada em torno de um conjunto reduzido de valores, gerando padrões mais regulares e aumentando a probabilidade de usar padrões já presentes no dicionário. Porém os modos de predição do H.264/AVC são limitados, e não apropriados para blocos com texturas mais complexas e não-homogêneas. Os valores na mesma direção devem seguir um padrão determinado por um conjunto de pixels da vizinhança do bloco, e apenas 9 possíveis direções podem ser escolhidas, não tendo qualquer flexibilidade para se ajustar a novas inclinações.

Com a finalidade de ultrapassar as limitações mencionadas, um novo modo de predição foi adicionado. O modo de predição baseado no critério dos mínimos quadrados, Leastsquare Prediction - LSP, determina um conjunto ótimo de coeficientes do filtro de predição para cada pixel do bloco, de acordo com o contexto local. Desta maneira a predição LSP é capaz de se adaptar as diversas condições locais, e proporcionar uma predição mais precisa onde os modos de predição direcionais do H.264/AVC falham.

A distribuição dos resíduos após a predição pode ser relacionada com uma distribuição de uma Gaussiana Generalizada [6], sendo determinada pelos seus parâmetros $\alpha$ (parâmetro de forma, descreve a taxa de decaimento da curva) e $\beta$ (parâmetro de escala, descreve o desvio padrão da distribuição). Distribuiçõos conhecidas como a distribuição uniforme, gaussiana ou laplaciana podem ser obtidas a partir da distribuição de uma gaussiana generalizada, fazendo $\alpha=\infty, \alpha=2$ e $\alpha=1$, respectivamente. Quanto mais concentradas forem as curvas, isto é, curvas com picos maiores e menor desvio padrão, mais similares serão os padrões dos resíduos. Isso facilita a adaptação do dicionário usado 
pelo MMP, uma vez que padrões já existentes no dicionário podem ser usados para aproximarem outros blocos da imagem, aumentando assim o poder de compressão do algoritmo.

Podemos ver o benefício de usarmos o LSP no formato das distribuições dos resíduos com a adição deste modo adaptativo de predição. No caso da imagem Barbara, se usarmos apenas os modos de predição do H.264, os parâmetros de distribuição da Gaussiana Generalizada que aproximam a sua distribuição são iguais a $\alpha=0.6870$ e $\beta=17.3961$. Já se adicionarmos o modo de predição adaptativo LSP, passamos a ter uma distribuição com um pico mais acentuado e menor desvio, como indicam os parâmetros da nova distribuição $\alpha=0.6727$ e $\beta=13.6212$, e também pode ser visto na Figura 1 .

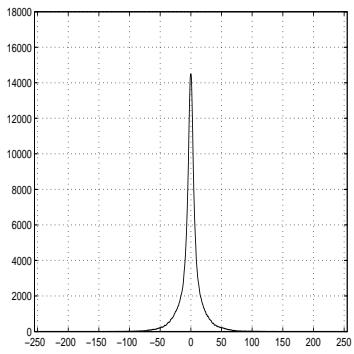

(a) Sem LSP

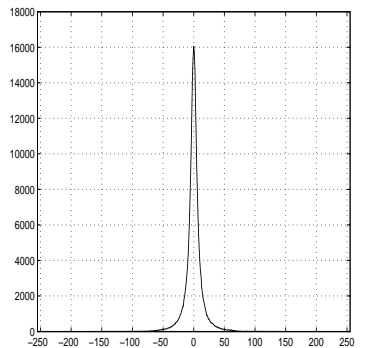

(b) Com LSP
Fig. 1. Distribuição de probabilidade do resíduo

\section{A. Predição baseado no critério dos mínimos quadrados}

O método de predição baseado no critério dos mínimos quadrados (LSP) filtra adaptativamente uma determinada vizinhança, onde os coeficientes do filtro são determinados usando uma janela de treinamento com os valores reconstruídos. A vizinhança é determinada de acordo com um modelo markoviano de ordem $\mathrm{N}$, e usualmente os pixels mais próximos são usados. A predição é obtida então com a seguinte equação:

$$
\hat{X}\left(\mathbf{n}_{0}\right)=\sum_{i=1}^{N} a_{i} X\left(\mathbf{n}_{i}\right)
$$

onde $a_{i}$ são os coeficientes do filtro de predição.

Note que na maioria dos casos os coeficientes indicam uma média ou o valor mediano da vizinhança, como sugere os modos de predição do H.264. No caso do LSP, esses coeficientes são otimizados localmente usando uma janela de treinamento causal seguindo o critério dos mínimos quadrados. Uma escolha adequada da janela de treinamento é uma área contendo dois retângulos de dimensões $T$ e $T+1$, totalizando $M=2 T(T+1)$ elementos (veja a Figura 2 ). A sequiência de treinamento será um vetor coluna

$$
\mathbf{y}=[X(n-1) \ldots X(n-M)]^{T}
$$

de tamanho $M \times 1$, e a vizinhança de predição forma uma matriz de tamanho $M \times N$, onde

$$
C=\left[\begin{array}{ccc}
X(n-1-1) & \ldots & X(n-1-N) \\
\vdots & & \vdots \\
X(n-M-1) & \ldots & X(n-M-N)
\end{array}\right]
$$

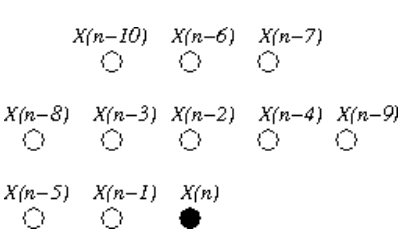

(a) Vizinhança do pixel

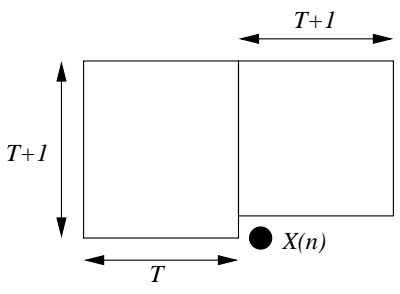

(b) Pixels de treinamento
Fig. 2. Implementação da predição em bloco usando o LSP

Os coeficientes do filtro de predição podem ser determinados por um processo de otimização baseado no critério dos mínimos quadrados, ao achar a solução para $\min \left(\|\mathbf{y}-C \mathbf{a}\|^{2}\right)$. Uma conhecida solução fechada para este problema é dado por

$$
\mathbf{a}=\left(C^{T} C\right)^{-1}\left(C^{T} \mathbf{y}\right)
$$

Um resultado equivalente pode ser obtido através da teoria de filtragem de Wiener [16]. Os coeficientes do filtro são obtidos através da minimização da função objetivo, definida pela média estatística do erro quadrático $\xi(k)=E\left[e^{2}(k)\right]=$ $E\left[\left(d-\mathbf{a}^{T} \mathbf{x}(k)\right)^{2}\right]$, onde o erro é definido como a diferença entre o valor desejado $d(k)$ e o valor estimado pela filtragem $\mathbf{a}^{T}(k) \mathbf{x}(k)$. Para um filtro com coeficientes fixos, a função de erro médio quadrático é dada por

$$
\xi=E\left[d^{2}(k)\right]-2 \mathbf{a}^{T} E[d(k) \mathbf{x}(k)]+\mathbf{a}^{T} E\left[\mathbf{x}(k) \mathbf{x}^{T}(k)\right] \mathbf{a}
$$

Os valores ótimos para os coeficientes do filtro que minimiza a função objetivo para um processo estacionário Gaussiano são obtidos zerando o gradiente da função acima. Este valores são determinados pela estatística de segunda ordem (matriz de covariância, $\left.R_{X X}=E\left[\mathbf{x}(k) \mathbf{x}^{T}(k)\right]\right)$ e pela estatística entre entrada e valor desejado (vetor de correlação cruzada, $\mathbf{r}_{X}=$ $E[d(k) x(k)])$.

$$
\mathbf{a}=R_{X X}^{-1} \mathbf{r}_{X}
$$

Esta solução também é conhecida como solução de Wiener. Porém na prática, estimativas precisas de $R_{X X}$ e $\mathbf{r}_{X}$ não estão disponíveis. Além do mais, em geral um modelo estatístico preciso para imagens não é nem estacionário nem Gaussiano. Uma maneira de estimarmos as estatísticas locais é aplicarmos o conceito de ergodicidade e usarmos as médias temporais em vez das médias estatísticas. Logo, $\left(\mathbf{r}_{X}, R_{X X}\right)$ podem ser calculados da seguinte forma

$$
R_{X X}=\frac{1}{M} C^{T} C, \quad \mathbf{r}_{X}=\frac{1}{M} C^{T} \mathbf{y}
$$

Ao substituirmos (5) em (4), obtemos novamente (2).

O LSP é particularmente eficaz em prever bordas de orientação arbitrária, devido a sua propriedade borda-orientada [13], onde os pixels da borda atuam de forma dominante na otimização dos mínimos quadrados. Se a janela de treinamento possui pixels de borda suficientes, a predição LSP pode identificar de forma eficiente a direção da borda e corretamente prever o valor do novo pixel. 


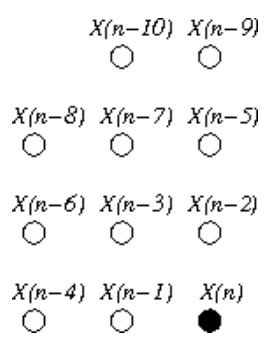

(a) Vizinhança causal

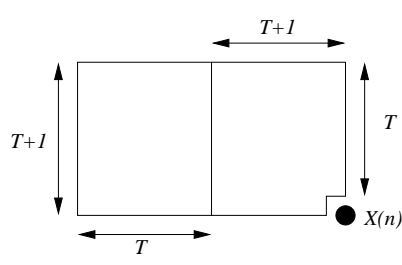

(b) Treinamento causal

Fig. 3. Modificações para a predição em blocos do LSP

\section{B. LSP aplicado a blocos}

Em [14], a predição de blocos usando o LSP é proposta. Em codificação com perdas, apenas os pixels reconstruídos estão disponíveis para serem utilizados na predição. Portanto, ao usarmos a predição de blocos, apenas os valores de pixels pertencendo a blocos acima e à esquerda do bloco atual é que podem ser utilizados no cálculo da predição do pixel, mesmo para aqueles que estão nas posições mais afastadas desta vizinhança.

$\mathrm{Na}$ nossa implementação, flexibilizamos a restrição da vizinhança e escolhemos sempre os pixels mais próximos para a predição. Nas posições que se encontram dentro do bloco e os coeficientes restaurados ainda não estão presentes, utilizamos o valor recém calculado na predição passada com o LSP para subtituir essas posições indisponíveis. A Figura 2 (a) mostra a vizinhança escolhida para a predição e a Figura 2 (b) ilustra os pixels usados no treinamento.

Como no caso da predição em bloco os pixels não são varridos na forma raster, nem sempre os pixels à direita e acima estão disponíveis para fazer o treinamento ou para serem usados na predição. Desta forma é necessário alterar os pixels usados para a predição e a janela de treinamento, com a finalidade de usarmos apenas pixeis causais, ou seja, que já foram determinados. As Figuras 3 (a) e (b) mostram a janela de treinamento alterada e a vizinhança usada nestes casos, onde elementos à direita não estão disponíveis. É importante notar que em ambos os casos (Figuras 2 e 3 ), teremos que usar em certas ocasiões os pixels preditos no lugar dos pixels decodificados nas posições dentro do bloco.

O LSP é um modo que depende da qualidade do treinamento realizado para estimar os coeficientes, portanto ele atua melhor em taxas mais altas, uma vez que a vizinhança disponível para o treinamento é mais fiel. O seu desempenho também irá variar de acordo com a imagem, onde imagens com bordas mais acentuadas, como a imagem Barbara, obtém ganhos maiores do que outras imagens com bordas mais suaves.

Como é usual na maioria dos algoritmos baseados em casamento de padrões, a complexidade computacional é afetada em sua maioria pela busca do índice ótimo do dicionário, sendo que o efeito da predição do bloco é desprezível. Porém no caso do LSP, é necessário efetuar uma operação de inversão de matrizes para cada pixel, o que tem um impacto não-desprezível na complexidade do algoritmo. Algumas implementações rápidas do LSP já foram propostas em [13]; no entanto, como este não é o foco da nossa abordagem mas

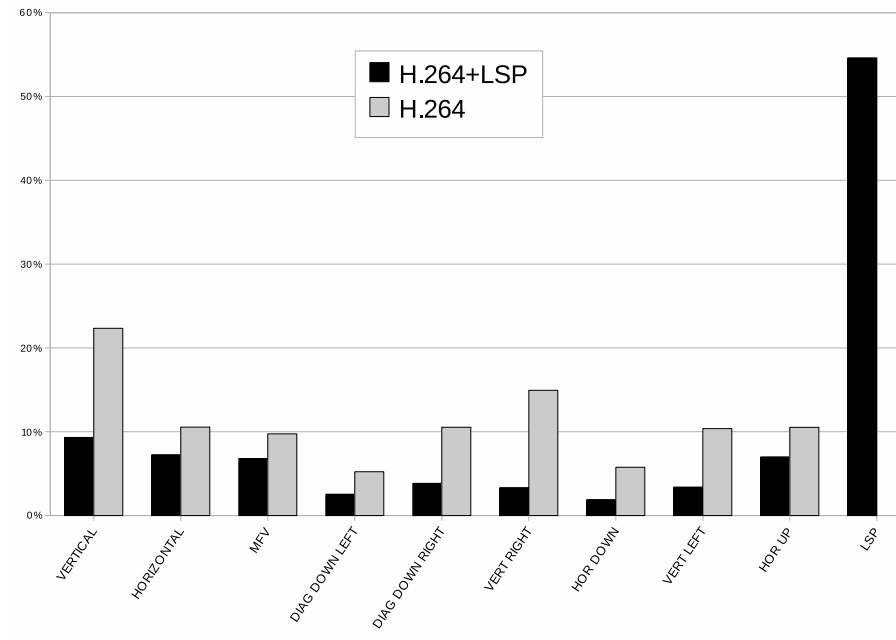

Fig. 4. Percentagem de escolha dos modos de predição para a imagem Barbara.

sim o desempenho taxa-distorção do algoritmo, esse tema não será considerado neste artigo, e será foco de estudos futuros.

\section{RESUltados EXPERIMENTAIS}

O principal motivação para usar o LSP era melhorar o desempenho das curvas de taxa-distorção do algoritmo MMP para imagens suaves e com muita textura, sem provocar perdas no comportamento eficiente do MMP relacionado com a codificação de imagens não-suaves. Nesta seção apresentamos os resultados experimentais, que justificam a utilização de mais um modo de predição adaptativo no esquema MMP. Além dos resultados do algoritmo proposto, os resultados de dois outros algoritmos estado-da-arte, o JPEG2000 e o codificador intra H.264/AVC High profile, também serão usados para efeito de comparação.

De maneira a explorar toda a capacidade de predição do modo LSP, um modelo de ordem alta foi utilizado, ou seja, uma vizinhança fixa de 10 elementos, conforme se indica nas Figuras 2 e 3 . O tamanho da janela de treinamento escolhido foi $T=7$. Estudos empíricos realizados em [13] sugerem que janelas maiores do que 7 não melhoram o desempenho do método de predição LSP.

$\mathrm{Na}$ Figura 4, vemos o número de vezes que cada modo de predição foi escolhido, com a configuração original e com os mesmos modos anteriores acrescidos do modo adaptativo, o LSP. Nota-se claramente a maior adoção do método LSP comparado com os outros métodos (mais de 50\%). O LSP irá ser usado especialmente em áreas onde a direção da imagem não corresponde exatamente às direções definidas pelos modos de predição H.264/AVC, e por conta disso vemos que modos diagonais são usados com menos freqüência na presença do LSP. No caso da figura Barbara, os padrões bem definidos da roupa irão usar modos diagonais e verticais, porém na presença do LSP esses modos são substituídos pelo modo adaptativo, resultando num resíduo para codificação de muito menor energia, e por consequiência de maior compressão.

Uma outra maneira de visualizarmos a escolha frequente do modo de predição LSP é também através da Figura 5, que 


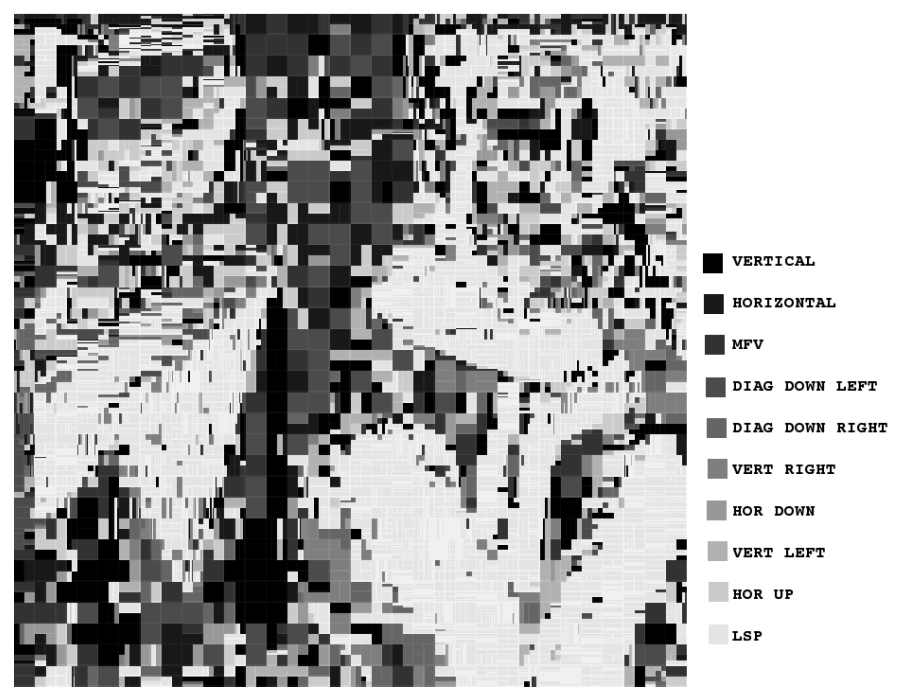

Fig. 5. Seleção dos modos de predição

mostra o mapeamento dos modos de predição. Nesta figura, cada nível de cinza representa um modo de predição diferente, e o nível mais claro indica o modo de predição LSP. Na Figura 6(a), podemos ver a imagem de predição obtida. Com estas figuras fica evidenciado que as áreas onde o LSP atuou mais fortemente foram as áreas da roupa da mulher e da toalha de mesa, áreas que apresentam padrões com bordas acentuadas. Nestas áreas, a presença das bordas nas janelas de treinamento contribui para a correta predição do valor do pixel, usando o critério dos mínimos quadrados.

Outras curvas de taxa distorção para imagens diferentes podem ser visualizadas nas Figuras 7(a) - 7(d). Outro fato marcante, já mencionado anteriormente, é que o ganho do LSP depende da imagem a ser codificada. Para imagens suaves com textura complexa, como a imagem Barbara, ganhos de até $1.2 \mathrm{~dB}$ foram registrados, enquanto que os ganhos para a imagem Lena são menores, em torno de $0.25 \mathrm{~dB}$. Podemos também notar que o desempenho das curvas melhora com taxas médias e altas. Uma vez que o modo LSP depende diretamente da janela de treinamento utilizada na predição do pixel, taxas mais altas que contém pixels reconstruídos mais precisos vão contribuir positivamente no treinamento. Para todas as imagens, podemos constatar o desempenho acima das curvas de taxa distorção dos algoritmos estado-da-arte usados para efeito de comparação, o JPEG2000 e o H.264/AVC.

Para imagem compostas e com textos, como as imagens PP1205 e PP1209 ${ }^{1}$, as bordas tem variações muito bruscas e ocorrem frequentemente, dificultando o processo de predição do LSP (ou de qualquer outro método), tornando difícil o aprendizado de uma borda dentro da janela de treinamento. Mesmo assim, a adição de mais um modo de predição não afetou o desempenho das curvas de taxa-distorção do algoritmo MMP para as imagens não-suaves, como podemos ver nas Figuras 7(c) e 7(d).

${ }^{1}$ Estas e outras imagens estão disponíveis para download através do site http://www.lps.ufrj.br/profs/eduardo/MMP

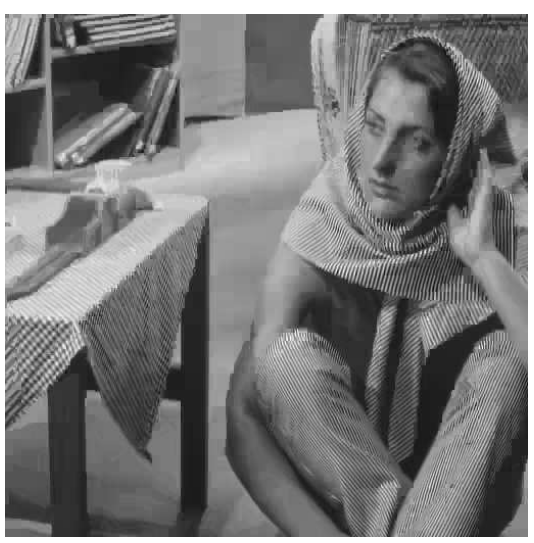

(a) Quadro com valores de predição $(25.448 \mathrm{~dB})$

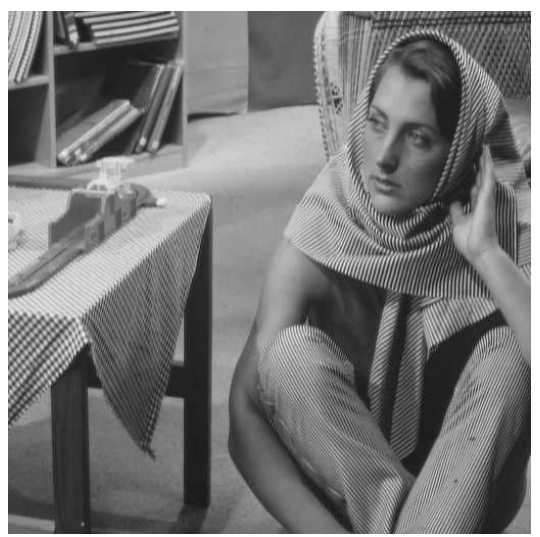

(b) Quadro decodificado (0.586 bpp - $34.784 \mathrm{~dB})$

Fig. 6. Visualização da predição LSP para a imagem Barbara com $\lambda=50$.

\section{CONCLUSÕES}

Neste artigo propomos o uso de um esquema de predição adaptativa baseado no critério dos mínimos quadrados (LSP) em conjunto com o algoritmo de casamento de padrões recorrentes em múltiplas escalas. A predição LSP foi adaptada para ser usada em blocos, e assim o codificador MMP passou a dispor de mais um método de predição, além dos usuais 9 métodos que já eram usados, derivados do padrão H.264/AVC.

A adição do modo de predição LSP permitiu ao algoritmo capturar as características locais da textura e através da estimação de coeficientes lineares, obter uma predição mais adequada de imagens com bordas acentuadas e direções arbitrárias. Pela sua grande eficiência, o modo LSP passa a ser mais utlizado que os diversos outros modos H.264/AVC existentes. Isso nos gera em certos casos ganhos de até $1 \mathrm{~dB}$ nas curvas de taxa-distoção para taxas médias e altas.

Além de ter melhorado o desempenho taxa-distorção do codificador MMP para imagens suaves, o modo LSP não apresentou qualquer perda de desempenho nas curvas de taxa-distorção para imagens não-suaves, incluindo gráficos e textos. Desta forma, o método MMP atinge uma qualidade objetiva superior a de codificadores estado-da-arte baseado em transformada para diversas taxas, em todos os tipos de imagens.

LSP se mostrou capaz de aumentar significativamente o desempenho das curvas de taxa-distorção do codificador MMP, 


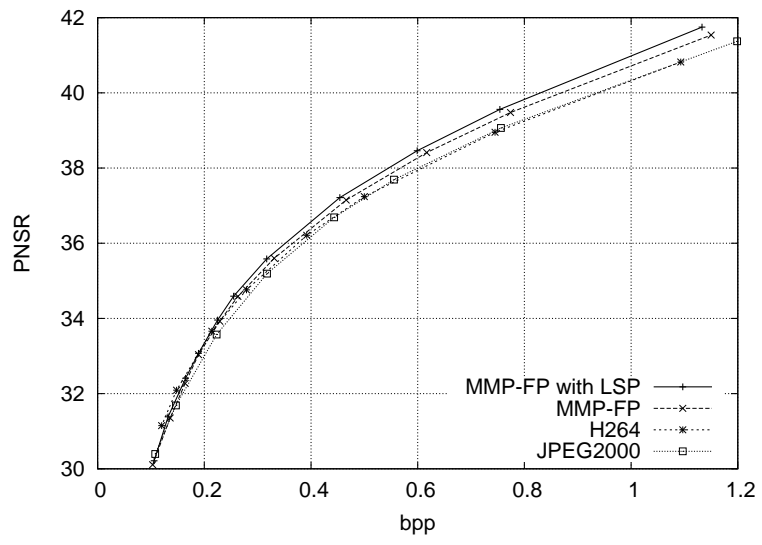

(a) Imagem Lena

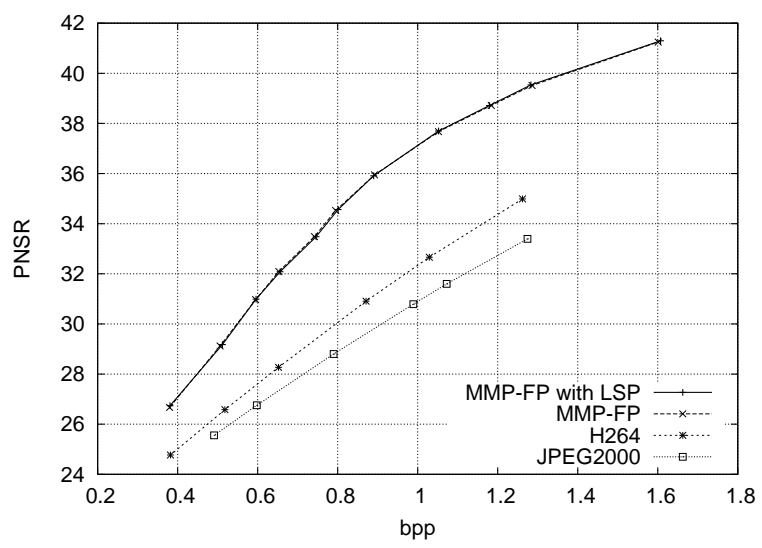

(c) Imagem PP1205

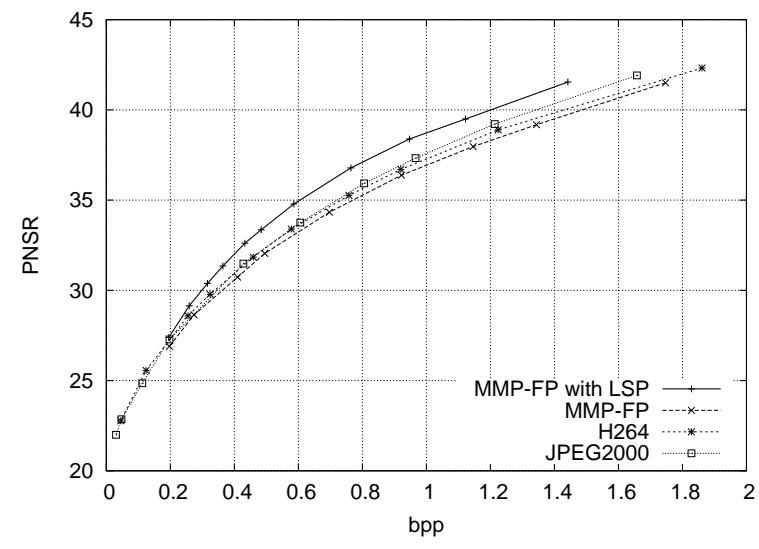

(b) Imagem Barbara

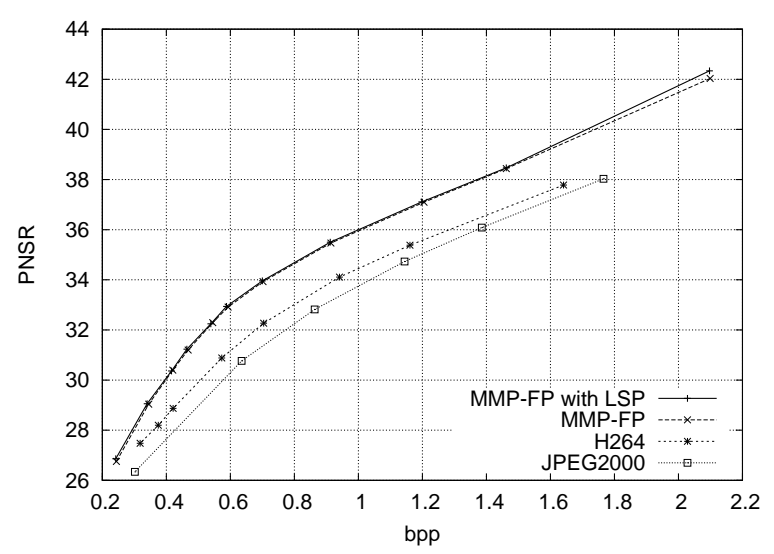

(d) Imagem PP1209

Fig. 7. Qualidade Objetiva (PSNR) para várias taxas de compressão

justificando a sua inclusão, apesar do aumento do custo computacional. Em trabalhos futuros, métodos alternativos para a estimação destes coeficientes de predição lineares visando diminuir a complexidade computacional da predição serão estudados. Algoritmos semelhantes, porém computacionalmente mais eficientes, como o RLS (Recursive Least Square) [16], serão levados em consideração.

\section{REFERÊNCIAS}

[1] Joint Video Team (JVT) of ISO/IEC MPEG \& ITU-T VCEG (ISO/IEC JTC1/SC29/WG11 and ITU-T SG16 Q.6), Draft of Version 4 of H.264/AVC (ITU-T Recommendation H.264 and ISO/IEC 14496-10 (MPEG-4 part 10) Advanced Video Coding), March 2005.

[2] T. Wiegand, G.J. Sullivan, G. Bjntegaard, and A. Luthra, "Overview of the H.264/AVC video coding standard," Circuits and Systems for Video Technology, IEEE Transactions on, vol. 13, no. 7, pp. 560-576, July 2003.

[3] M. de Carvalho, E. da Silva, and W. Finamore, "Multidimensional signal compression using multiscale recurrent patterns," Elsevier Signal Processing, vol. 82, pp. 1559-1580, November 2002.

[4] D. S. Taubman and M.W. Marcelin, JPEG2000: Image Compression Fundamentals, Standards and Practice, Kluwer Academic Publishers, 2001.

[5] N. M. M. Rodrigues, E. A. B. da Silva, M. B. de Carvalho, S. M. M. de Faria, and Vitor M. M. Silva, "Universal image coding using multiscale recurrent patterns and prediction," IEEE International Conference on Image Processing, September 2005.

[6] N. M. M. Rodrigues, E. A. B. da Silva, M. B. de Carvalho, S. M. M. de Faria, and Vitor M. M. Silva, "On dictionary adaptation for recurrent pattern image coding," Image Processing, IEEE Transactions on, vol. 17, no. 9, pp. 1640-1653, September 2008.
[7] Nelson C. Francisco, N. M. M. Rodrigues, E. A. B. da Silva, M. B. de Carvalho, S. M. M. de Faria, Vitor M. M. Silva, and Manuel J. C. S. Reis, "Casamento aproximado de padrões multiescala com segmentação flexível e treino do dicionário," Simpósio Brasileiro de Telecomunicações, September 2008.

[8] Yung Lyul Lee, Ki-Hun Han, and G. J. Sullivan, "Improved lossless intra coding for h.264/mpeg-4 avc.," IEEE Transactions on Image Processing, vol. 15 , no. 9, pp. 2610-2615, 2006.

[9] Zhang Xiong-Wei Huang Hui, Cao Tie-Yong, "The enhanced intra prediction algorithm for H.264," IEEE International Conference on Image Processing, October 2008.

[10] K. Sugimoto, M. Kobayashi, Y. Suzuki, S. Kato, and C. Seng Boon, "Inter frame coding with template matching spatio-temporal prediction," IEEE International Conference on Image Processing, October 2004.

[11] N. M. M. Rodrigues, E. A. B. da Silva, M. B. de Carvalho, S. M. M. de Faria, and Vitor M. M. Silva, "Multiscale recurrent pattern predictive image coding with template matching," Conference on Telecommunications - ConfTele07, May 2007.

[12] Xin Li, "Least-square prediction for backward adaptive video coding," EURASIP J. Appl. Signal Process., vol. 2006, no. 1, pp. 126-126, 2006.

[13] Xin Li and Michael T. Orchard, "Edge-directed prediction for lossless compression of natural images," IEEE Transactions on Image Processing, vol. 10, no. 6, pp. 813-817, 2001.

[14] Limin Liu, Yuxin (Zoe) Liu, and Edward J. Delp, "Enhanced intra prediction using context-adaptive linear prediction," PCS2007 - Picture Coding Symposium, November 2007.

[15] Nelson C. Francisco, N. M. M. Rodrigues, E. A. B. da Silva, M. B. de Carvalho, S. M. M. de Faria, Vitor M. M. Silva, and Manuel J. C. S. Reis, "Multiscale recurrent pattern image coding with a flexible partition scheme," IEEE International Conference on Image Processing, October 2008.

[16] Paulo S. R. Diniz, Adaptive Filtering Algorithms and Practical Implementation, Kluwer Academic Publishers, 2002. 